\title{
Wild-type blocking pcr coupled with internal competitive amplified fragment improved the detection of rare mutation of $K R A S$
}

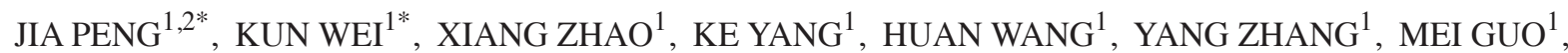 \\ JING HE $^{1}$, HAIYAN WU ${ }^{1}$, YONGCHUAN LI ${ }^{1}$, NA ZHAO ${ }^{1}$, QING HUANG $^{1}$ and WEILING FU ${ }^{1}$ \\ ${ }^{1}$ Department of Laboratory Medicine, Southwest Hospital, The Third Military Medical University, Chongqing 400038; \\ ${ }^{2}$ Department of Laboratory Medicine, 324th Hospital of PLA, Chongqing 400020, P.R. China
}

Received February 2, 2017; Accepted June 8, 2017

DOI: $10.3892 / \mathrm{mmr} .2017 .6883$

\begin{abstract}
Mutant KRAS proto-oncogene GTPase (KRAS) serves an important role in predicting the development, diagnosis, treatment and efficacy of targeted drug therapies for colorectal cancer. To improve the detection efficacy of trace amount of mutant $K R A S$, the locked nucleic acid-based method was modified in the present study. Internal competitive amplification fragments were used to improve the inhibition of wild-type $K R A S$ with a wild-type blocking (WTB) probe and specifically amplify the trace amounts of mutant KRAS. The modified method, quantitative clamp-based polymerase chain reaction technology using WTB coupled with internal competitive reference to enhance the amplification specificity, named WIRE-PCR, completely blocked the amplification of wild-type KRAS in 50-150 ng DNA templates. The added internal competitive amplified fragments were amplified together with the target gene, which were used to reduce base mismatch due to the high number of cycles in PCR and quantify the total amount of DNA. The results demonstrated that WIRE-PCR facilitated the detection of mutated alleles at a single molecular level. In the colorectal biopsies from 50 patients with suspected colorectal cancer, 18 cases (36\%) contained mutant KRAS, and the amount of mutant DNA accounted for $18.6-64.2 \%$ of the total DNA. WIRE-PCR is a simple, rapid and low-cost quantitative analysis method for the detection of trace amounts of the mutant KRAS.
\end{abstract}

Correspondence to: Professor Weiling Fu or Professor Qing Huang, Department of Laboratory Medicine, Southwest Hospital, The Third Military Medical University, 30 Gaotanyan Street, Chongqing 400038, P.R. China

E-mail: weiling_fu@163.com

E-mail: drq.huang@gmail.com

*Contributed equally

Key words: KRAS gene, colorectal carcinoma, locked nucleic acid, $\mathrm{PCR}$, internal competitive amplification fragments

\section{Introduction}

Colorectal carcinoma (CRC) is one of the highest incident malignant tumors in the world. The five-year survival rate is less than $10 \%$, and $50-60 \%$ of the CRC patients eventually progress to metastatic colorectal carcinoma (mCRC) $(1,2)$. Although FOLFIRI, FLOFLOX, and other chemotherapy alone or in combination with anti-VGFR monoclonal antibody (e.g., Bevacizumab) can improve the prognosis of patients with $\mathrm{mCRC}$, who may produce drug resistance, application of epidermal growth factor receptor (EGFR) monoclonal antibody (e.g., Panitumumab) at the moment is still effective $(3,4)$. As a member of ErbB transmembrane tyrosine kinase receptor family, EGFR activates MEK/ERK, PI3K/AKT, and STAT signaling pathways to induce cell proliferation, dedifferentiation and blocking of apoptosis (5). Anti-EGFR monoclonal antibodies, mainly include Cetuximab and Panitumumab, have been used in the clinical therapy of mCRC (6). KRAS is a downstream EGFR-signaling pathway-core component of MAPK pathway. Previous studies have shown that its $K R A S$ mutation leads to failure of Erbitux, Panini, or other monoclonal antibody therapy due to ineffective inhibition of EGFR signaling pathway using those monoclonal antibodies, indicating that mutant KRAS is critical for EGFR monoclonal and targeted antibody therapy (7). The European Medicines Agency requires clinicians to detect KRAS mutation in the patients before administrating monoclonal antitumor drug (8).

A variety of detection methods have been available for the screening of mutant $K R A S$, including sequencing, single-stranded confirmation polymorphism (SSCP), AS-PCR, TaqMan probe PCR, beads, emulsion, amplification, and magnetics (BEAMing), LigAmp assay, and clamping-based PCR $(9,10)$. Clamping-based PCR is the most sensitive method for the detection of low abundance mutations by selectively adding the wild-type amplified nucleic acid into the reaction system to block the amplification of wild-type gene $(11,12)$. The related techniques have been successfully applied to detect the trace amount of gene mutation in some tumors $(12,13)$. However, the application of clamping-based PCR also has a problem: Susceptible to the interference of DNA polymerase in the reaction system, which may result 
in base mismatch in PCR with high cycle number (14-16). To solve this problem, the present study modified the existing clamping-based PCR by adding internal competitive amplified fragments to enhance the inhibition of wild-type KRAS via locked nucleic acid (LNA) probe and established a method for detecting the trace amount of mutant KRAS in colorectal neoplasms. The method was applied to detect mutant KRAS from the colorectal biopsies of 50 patients with suspected colorectal cancer, followed by DNA sequencing and pathological analysis to validate the test results. The present study provided a reference for effectively predicting the therapeutic outcomes of colorectal cancer patients through our methods on mutant KRAS detection.

\section{Materials and methods}

Patients and DNA extraction from colorectal biopsies. The present study recruited Han Chinese patients from the outpatient and inpatient clinics of the Southwest Hospital (Chongqing City, China). Our study protocol was approved by the Ethics Committee of the Southwest Hospital. All patients or their guardians signed the informed consents before participating in the present study.

Fresh colorectal biopsies obtained during colonoscopy from the patients with suspected colorectal cancer were washed in PCR and subsequently placed in new Eppendorf tubes, followed by DNA extraction using Tissue DNA kit (Catalogue no. 536-050, Gene Tech Biotechnology Co., Ltd., Shanghai, China) with reference to the manufacturer's instructions and DNA quantification using Nanodrop to obtain mean value of total DNA of each sample from the triplicated measurements.

Amplification of mutant KRAS using WIRE-PCR. As shown in Table I, the PCR system in the present study contained $500 \mathrm{nM}$ primer set (SW-329/330) and $100 \mathrm{nM}$ florescent probe (SW-1294) for the detection of internal reference gene; $500 \mathrm{nM}$ primer set (SW-1595/1596) and $250 \mathrm{nM}$ fluorescent probe (SW-1438) for the detection of KRAS; and $500 \mathrm{nM}$ LNA probe (SW-144). The PCR conditions were $50^{\circ} \mathrm{C}$ for $2 \mathrm{~min}$ and 60 cycles of $95^{\circ} \mathrm{C}$ for $2 \mathrm{~min}, 95^{\circ} \mathrm{C}$ for $15 \mathrm{sec}$, and $60^{\circ} \mathrm{C}$ for $1 \mathrm{~min}$. The $2 \mathrm{X}$ SuperMix-UDG was used as the enzyme for the PCR system. To determine the sensitivity of WIRE-PCR system, a concentration gradient of mutant template was prepared by mixing increasing concentrations of plasmid $\left(10^{5}, 10^{4}, 10^{3}, 10^{2}\right.$, $10^{1}, 10^{0}$, copies $/ \mu 1$ ) from the previously constructed containing mutated single nucleotides (c34G $>$ C;G12R.1R) with human WT-gDNA in total $50 \mathrm{ng}$. The reaction conditions were $50^{\circ} \mathrm{C}$ for $2 \mathrm{~min}$ and 60 cycles of $95^{\circ} \mathrm{C}$ for $2 \mathrm{~min}, 95^{\circ} \mathrm{C}$ for $15 \mathrm{sec}$, and $60^{\circ} \mathrm{C}$ for $1 \mathrm{~min}$ in total $20 \mu \mathrm{l}$.

\section{Results}

Establishment and optimization of WIRE-PCR. Given the importance of the annealing temperature in PCR system, we optimized the best anneal temperature for the primer sets used in the present study. For example, anneal temperatures for the amplification of $K R A S$ gene using SW-1595/1596 primers were set as $60-68^{\circ} \mathrm{C}$ to amplify the PCR products at eight gradient temperatures. The results of agarose gel electrophoresis separating the PCR products of different annealing temperatures,
Table I. Sequences of oligonucleotides used in the present study.

\begin{tabular}{ll}
\hline Oligo ID & \multicolumn{1}{c}{ Oligo sequences (5'-3') } \\
\hline SW-329 & CAGTCTCCTCCAAACAGAAAGTCA \\
SW-330 & GTCCATCTTGGATAAGGTCAGGA \\
SW-1294 & (Texas Red) CGGTTTGGACTTCATTCCTGG \\
& GCTCC (BHQ2) \\
SW-1595 & TTTATTATAAGGCCTGCTGAAAATGAC \\
SW-1596 & CGTCAAGGCACTCTTGCCTAC \\
SW-1438 & (VIC) ACTACCACAAGTTTATATTC (MGB) \\
SW-144 & TACG $\underline{\text { TCACCAGCT }}$
\end{tabular}

${ }^{1}$ SW-329 and SW-330 were the primer sets of the internal reference LEPTIN; SW-1294 was the fluorescent probe of internal reference LEPTIN; SW-1595 and SW-1596 were the primer sets of the KRAS gene; and SW-1438 was the fluorescent probe of KRAS. The underlined letters in SW-144 indicate the LNA.

and the results showed the optimal temperature $60^{\circ} \mathrm{C}$, with good amplification efficacy and the corresponding annealing, which was suggested to be the optimal annealing temperature for the reaction system.

The mean CT values \pm standard deviation in the internal reference gene, $L E P T I N$-involved amplification reaction using 500 and $250 \mathrm{nM} K R A S$ primer set were $25.6 \pm 0.23$ and $26.52 \pm 0.36$, respectively. The small CT value of the reaction system using $500 \mathrm{nM} K R A S$ primer set showed good reproducibility, and thus we used $500 \mathrm{nM} K R A S$ primer set to optimize the KRAS amplification. Subsequent test using different concentrations of fluorescent probe of the internal reference gene (i.e., 50, 100, and $200 \mathrm{nM}$ ) showed that the wild-type blocking (WTB) probe in our WIRE-PCR using different concentrations of fluorescent probe of the internal reference gene could effectively block the amplification of wild-type KRAS. With reference to the results of LEPTIN and KRAS amplifications, the mean CT values of LEPTIN amplification group and $K R A S$ amplification group were relatively large when using $200 \mathrm{nM}$ fluorescent probe of the internal reference gene (Table II). Therefore, $50 \mathrm{nM}$ and $100 \mathrm{nM}$ fluorescence probe for the internal reference gene were considered to be the optimal concentrations in the system. Application of $100 \mathrm{nM}$ fluorescent probe of the internal reference gene better enhanced the fluorescence intensity of the KRAS amplification than the other two concentrations of the probe. In addition, the fluorescence signal was stable. Therefore, a final concentration of $100 \mathrm{nM}$ fluorescent probe of the internal reference gene was selected to optimize the $\mathrm{PCR}$ reaction, which contained $500 \mathrm{nM}$ primer set (SW-329/330) and $100 \mathrm{nM}$ florescent probe (SW-1294) for the detection of internal reference gene, LEPTIN; and $500 \mathrm{nM}$ primer set (SW-1595/1596) and $250 \mathrm{nM}$ fluorescent probe (SW-1438) for the detection of KRAS.

Blocking effect of different concentrations of wild-type template in the reaction system. The concentrations of most DNA samples extracted from the clinical biopsies ranged from 50 to $150 \mathrm{ng}$. Evaluation if the WTB concentration used in 
Table II. Comparison of the mean CT values of the LEPTIN and KRAS amplification groups.

\begin{tabular}{lccc}
\hline Mean CT value $\pm(\mathrm{SD})$ & Fluorescent probe $50 \mathrm{nM}$ & Fluorescent probe $100 \mathrm{nM}$ & Fluorescent probe 200 nM \\
\hline LEPTIN (with WTB) & $24.4 \pm 0.24$ & $24.33 \pm 0.68$ & $26.15 \pm 0.15$ \\
LEPTIN (without WTB) & $23.75 \pm 0.46$ & $23.81 \pm 0.76$ & $25.62 \pm 0.27$ \\
KRAS (with WTB) & $\mathrm{NA}$ & $\mathrm{NA}$ & $\mathrm{NA}$ \\
KRAS (without WTB) & $25.55 \pm 0.23$ & $25.68 \pm 0.55$ & $26.22 \pm 0.21$ \\
\hline
\end{tabular}

Table III. Blocking effects of different concentrations of wild-type template in the constructed wire PCR system.

\begin{tabular}{lcccc}
\hline Mean CT value $\pm(\mathrm{SD})$ & Template $50 \mathrm{ng} / \mu 1$ & Template $100 \mathrm{ng} / \mu 1$ & Template $150 \mathrm{ng} / \mu \mathrm{l}$ & Template 200 ng/ $\mu 1$ \\
\hline LEPTIN (with WTB) & $24.46 \pm 0.63$ & $23.75 \pm 0.20$ & $24.21 \pm 0.25$ & $23.54 \pm 0.2$ \\
LEPTIN (without WTB) & $24.07 \pm 0.08$ & $23.41 \pm 0.11$ & $24.86 \pm 0.15$ & $23.21 \pm 0.13$ \\
KRAS (with WTB) & NA & NA & NA & $36.78 \pm 0.61$ \\
KRAS (without WTB) & $24.16 \pm 0.90$ & $23.49 \pm 0.60$ & $22.75 \pm 0.11$ & $22.64 \pm 0.35$ \\
\hline
\end{tabular}

the reaction system could effectively block the amplification of different amounts of DNA templates was necessary at the early experimental stage. Different concentrations of DNA template (i.e., 50, 100, 150, and $200 \mathrm{ng} / \mu \mathrm{l}$ ) used in the KRAS amplification group imparted an effective blocking effect on 50-200 ng of the wild-type template under the WTB reaction. Non-specific amplification of KRAS was found in the system using $200 \mathrm{ng} / \mu \mathrm{l}$ template and near 40 cycles (with the mean CT value of $36.78 \pm 0.61$ ), indicating that application of $500 \mathrm{nM}$ WTB probe did not completely block the amplification of wild-type gene when using $200 \mathrm{ng}$ wild-type template (Table III and Fig. 1A and B). No significant difference of the mean CT values of LEPTIN amplification was found before and after adding WTB $(25.39 \pm 0.21$ and $25.37 \pm 0.04$, respectively $\mathrm{P}>0.05)$, suggesting that WTB had no significant effect on LEPTIN amplification (Fig. 1C and D). The results with and without WTB when added LEPTIN showed the internal amplified fragment were amplified together with the target gene, which were used to reduce base mismatch due to high number of cycles in PCR and enhanced the specificity (Fig. 1E and F).

The sensitivity capabilities of the WIRE-PCR system. According to the blocking efficiency curves in Fig. 2, we used the concentration gradient $\left(10^{5}, 10^{4}, 10^{3}, 10^{2}, 10^{1}, 10^{0}\right.$, copies $/ \mu 1$ ) plasmid (c34G $>$ C;G12R.1R) mixed with WT-gDNA in total $50 \mathrm{ng}$ as template to assessment the sensitivity. The result shown in Fig. 4 indicated that in the WIRE-PCR system, different concentration result in different $C_{q}$ values even at the level of single base pair of Plasmid. However, a previous study showed that traditional PCR always lead to consistent $\mathrm{C}_{\mathrm{q}}$ values despite the differences in the mutated template concentrations associated with the KRAS MT-alleles because of both WT-alleles and MT-alleles could be equivalently amplified (17). We used the $\mathrm{C}_{\mathrm{q}}$ values associated with traditional PCR to indicate the total quantity of input DNA in previous research. When WIRE-PCR utilized, the $\mathrm{C}_{\mathrm{q}}$ values increased with the quantity of KRAS mutation template, indicating that the amplification of KRAS WT-alleles was efficiently inhibited. And we added internal competitive amplified gene, not only can it amplified together with the target gene, but can used to reduce base mismatch due to high number of cycles in PCRs and quantify the total amount of DNA. The result showed the high blocking efficiency and indicated that the WIRE-PCR promotes the detection of KRAS MT-alleles with high sensitivity even at single base pair level (Fig. 2).

Based on the amplification curves in Fig. 2, we concluded that the WIRE-PCR was capable of detecting a single copy of KRAS MT-allele in high presence of WT-alleles with an amplification efficiency of $93.8 \%$ and $R^{2}=0.998$ (Fig. 3). The linear association suggest the amount of MT-alleles in a given sample could be quantified by the real-time PCR standard curve.

The race amount of mutant KRAS in the clinical biopsies. In the present study, WIRE-PCR was used to detect the trace amount of mutant KRAS in 50 colorectal biopsies collected during colonoscopy and find 18 positive cases, indicating that approximately $36 \%$ of the colorectal biopsies from the 50 patients had trace amount of mutant KRAS (18). Fig. 4A shows a colorectal biopsy that harbored a mutant $K R A S$ detected by our early constructed system. The green dashed line shows continued amplification of mutant KRAS after adding LNA, indicating that gene mutation in $K R A S$ occurred. Fig. 4B shows no specific gene amplification after adding LNA, suggesting no mutant KRAS is contained in that particular sample. Moreover, the red lines in Fig. 4A indicated that the amplification of internal competitive fragment was not affected before and after the addition of LNA, indicating a good reproducibility of our experimental results. To further confirm the mutation in 18 colorectal biopsies, we sent the PCR products of these 18 cases with positive $K R A S$ mutation for sequencing a biotechnology company in Shanghai, China. The results showed that $18 \mathrm{spec}-$ imens contained KRAS gene mutation. As shown in Fig. 4C and $D$, the green peak demonstrates the $G$ to A mutation, which was consistent with the immunohistochemical analysis 

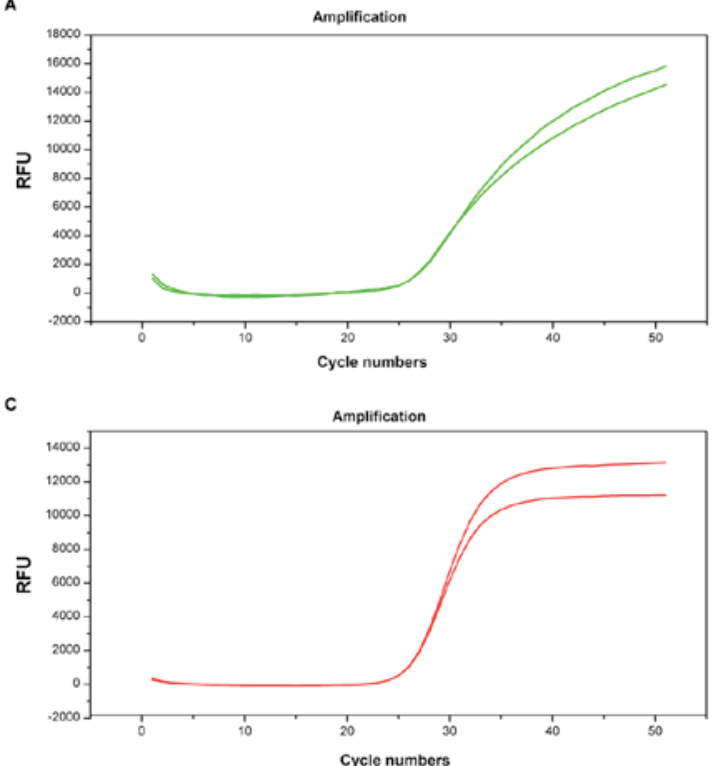

E

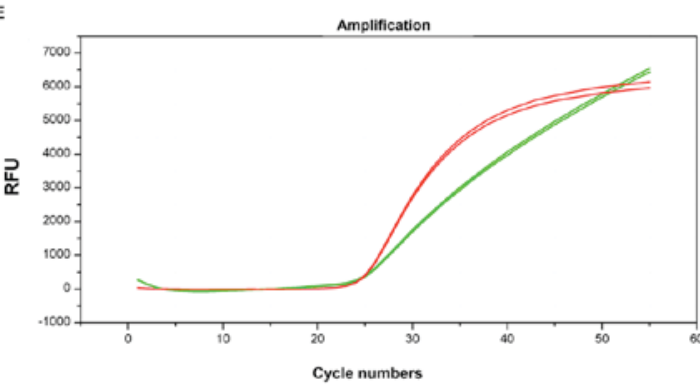

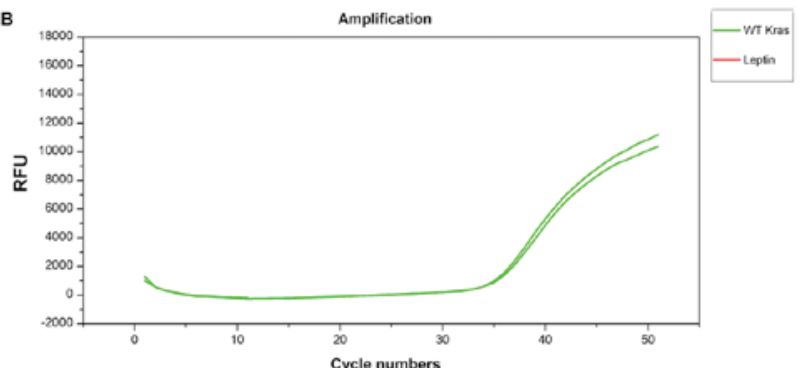
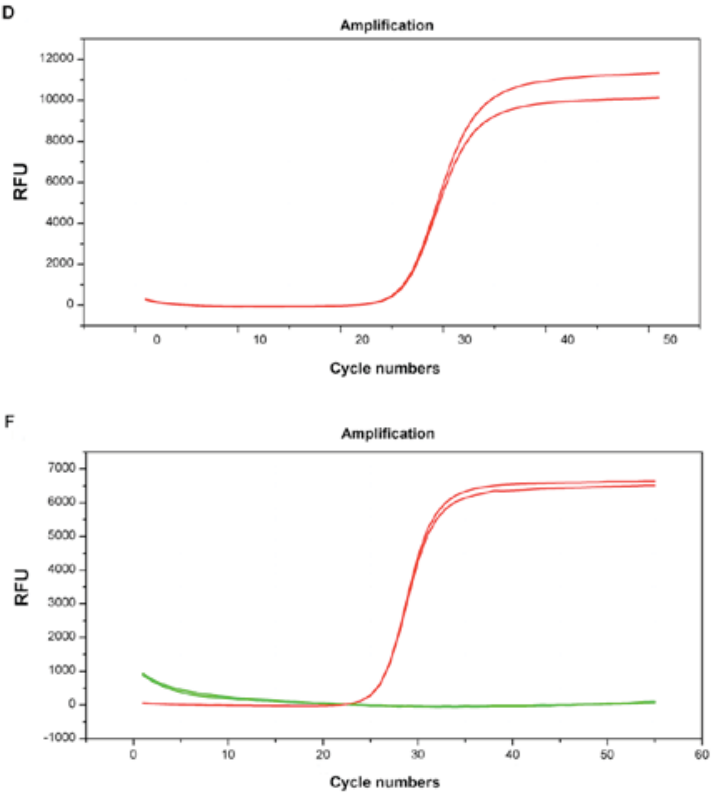

Figure 1. Blocking effects of the constructed WIRE-PCR system on wild-type template. Amplification curves of $L E P T I N$ and KRAS. Green line represents $K R A S$ and red represents $L E P T I N$. (A) Wild-type KRAS without WTB LNA probe. (B) Wild-type with WTB LNA probe. (C and D) $L E P T I N$ without and with WTB LNA probe. (E and F) WIRE-PCR system without and with WTB LNA probe. Addition of WTB LNA resulted in LEPTIN amplification only (red line) but a complete blockage of wild-type KRAS amplification. NTC were not found in amplification curves. WTB, wild-type blocking; LNA, locked nucleic acid.

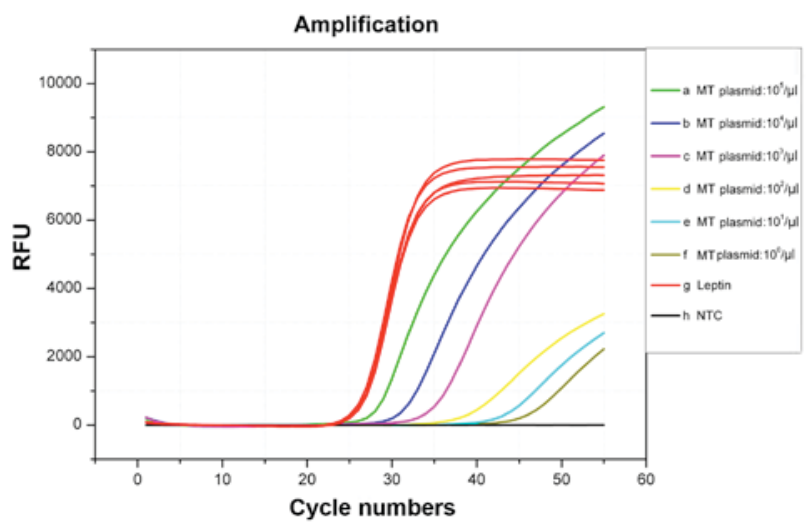

Figure 2. Sensitivity of real-time WIRE-PCR with internal competitive amplified fragment. Curves a to f show the amplification curves of real-time PCR with the WTB probe. Specified concentrations of template (MT-KRAS plasmid mixed with human WT-gDNA) indicated in each curve. LEPTIN gene as internal competitive amplified fragment in curve $\mathrm{g}$. The amplification completed in a $20 \mu 1$ reaction mixture. KRAS Plasmid (c34G $>$ C;G12R.1R) were diluted in different concentrations $\left(10^{5}, 10^{4}, 10^{3}, 10^{2}, 10^{1}, 10^{0}\right.$, copies $\left./ \mu 1\right)$ spiked into samples containing WT-gDNA. WTB, wild-type blocking.

of the corresponding specimens under 20x magnification of light microscopy. Calculation of the changes of CT values of KRAS amplification group before and after the addition

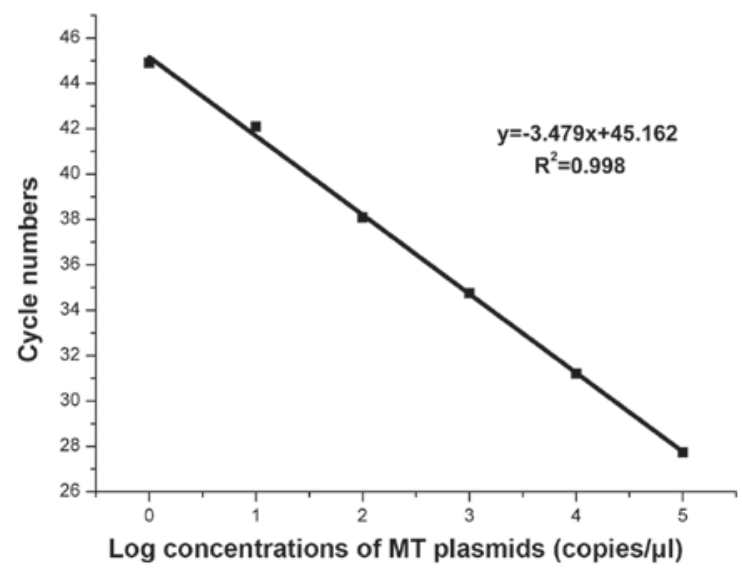

Figure 3. Quantitative curves of real-time WIRE-PCR. Standard curve was generated by plotting the average $\mathrm{C}_{\mathrm{q}}$ values from real-time PCR against the $\log$ concentrations of mutant KRAS plasmid. The amplification efficiency of real-time WTB-PCR was $93.8 \%$ (slope, $-3.479 ; \mathrm{R}^{2}=0.998$ ). WTB, wild-type blocking.

of LNA as well as the CT values of the internal competitive amplified fragment, LEPTIN, helped assess the KRAS mutation rate in each positive sample. Among the 18 biopsies with positive mutations, the KRAS mutation rate ranged from 18.6 
A

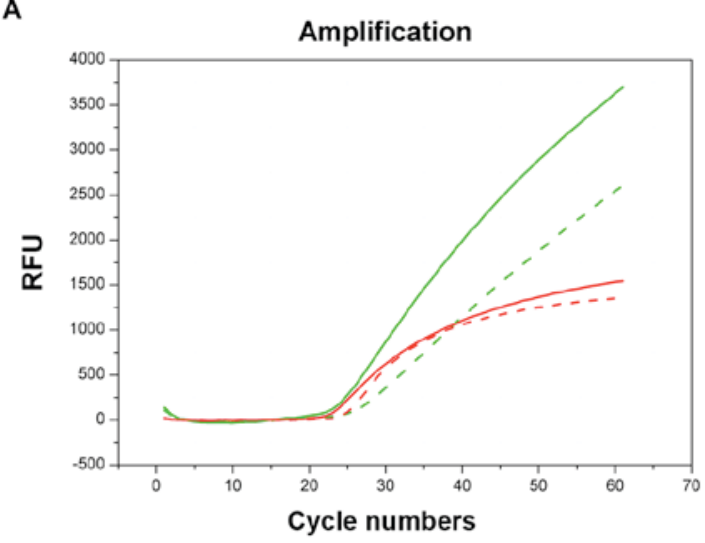

C

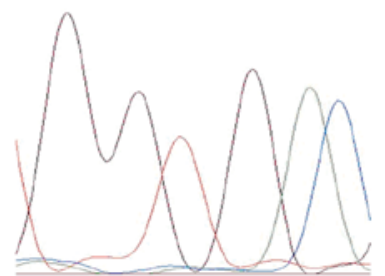

B

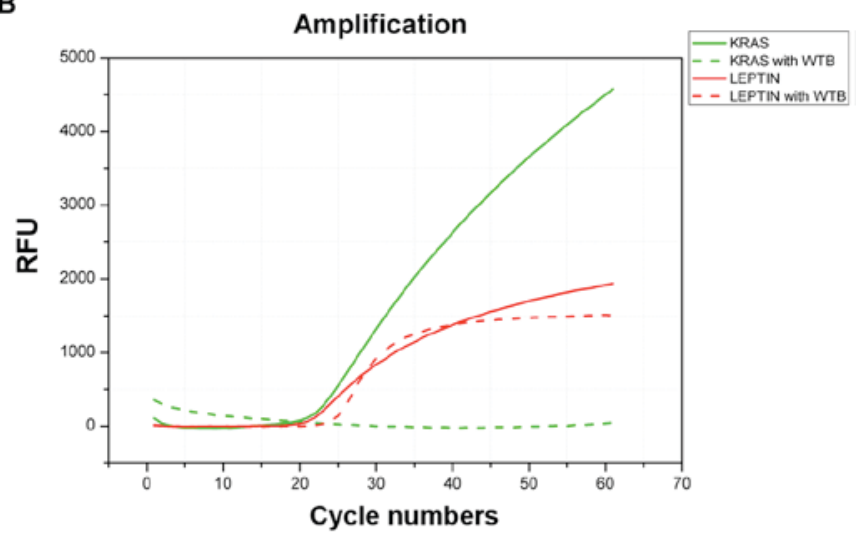

D

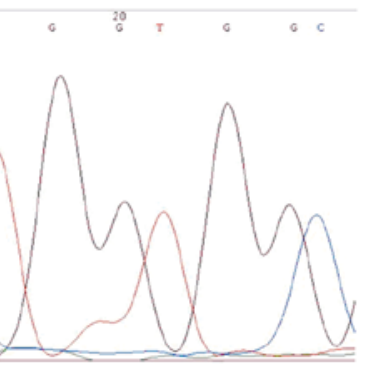

Figure 4. Detection of the trace amount of mutant KRAS in the clinical biopsies of colonoscopy. Panels (A and C) show the detection and amplification of trace amount of mutant $K R A S$. Panels (B and D) indicate no KRAS mutation. Green and red lines represents KRAS and internal amplified fragment (LEPTIN) respectively, Dash lines indicate WTB LNA probe were added in the reaction. NTC were not found in amplification curves. WTB, wild-type blocking; LNA, locked nucleic acid.

to $64.2 \%$. In the present study, the constructed WIRE-PCR effectively detected the trace amount of mutant $K R A S$ in the clinical biopsies.

\section{Discussion}

Different proportions of KRAS mutation have been discovered in a variety of human malignancies, such as malignant melanoma, lung cancer, colorectal cancer, and thyroid cancer. The KRAS mutation rate in the patients with rectal cancer is approximately $40 \%$ that included the point mutations in codons $12,13,15,18,61,117$, and 146 of exon 2, in which, the point mutations in codons 12 and 13 of exon 2 were common, accounting for approximately $40 \%(19,20)$. Mutant KRAS has become one of the markers affecting the prognosis of the colorectal cancer patients, and detection of mutant $K R A S$ is particularly important $(21,22)$. In a large number of detection assays for gene mutation, clamp-based PCR has given us good inspiration. Application of peptide nucleic acid (PNA) or LNA forms LNA/DNA chimeras, which closely bind with wild-type template and prevent the amplification of the wild-type template based on the high affinity binding between LNA/PNA and DNA (23). In clinical practice, LNA has been used as a substitute for PNA. According to the high affinity binding between LNA and DNA, LNA probe has been used to inhibit the wild-type PCR amplification (16). The principle is that the design of upstream and downstream primers is outside the LNA/DNA chimeras. In this reaction system, the polymerase lacking $5^{\prime}$ to $3^{\prime}$ end exonuclease activity ensured that LNA/DNA chimera probes were not hydrolyzed in the reaction system and LNA/DNA chimera probes were used for the inhibition of wild-type gene (24). To target the design of complementary oligonucleotide of sense strand $K R A S$, the present study added WTB probe into the reaction system. The added WTB probe sequence was partially overlapped with the wild-type template. KRAS and WTB probes competitively bound to the wild-type template in the same reaction system. Binding efficiency between LNA of the WTB probe and template was higher, occupying the base complementary binding region of primers and templates, thereby interfering the binding between primers and wild-type templates and inhibiting the amplification of the wild-type gene (11). In the $20 \mu \mathrm{l}$ PCR system, the addition of polymerase and bases is often superfluous and necessary for common PCR amplification reactions, However, non-targeted mutant gene amplification occurred between LNA/DNA chimeras and template binding region in the WTB-involved PCR leads to false positive results. With increasing number of PCR cycles, the products of non-targeted mutant gene amplification are continuously increased, which seriously affects the reading in the detection of trace amount of the single-point mutant gene.

In our previous study, we found that the KRAS of the wild-type samples also amplified under the WTB reaction when PCR system near high cycles. Under the thermodynamic driving force of DNA polymerase, the single base terminal mismatch between primers and template could easily trigger the non-specific amplification of an input DNA having opposite genotype (e.g., WT genotype) $(25,26)$. Moreover, weak-destabilization effects of terminal mismatches could 
further promote non-specific amplification (27). Although stringent reaction conditions can be used to dramatically reduce or eliminate non-specific amplification, boptimization is time-consuming, and sometimes unsuccessful. Internal reference gene added as internal competitive amplified fragments in the reaction system consumed any excess DNA polymerase and free base fragments, thereby enhancing the blocking effect of WTB probe on wild-type KRAS template and improving the detection efficiency for trace amount of $K R A S$ gene. In addition, since the internal competitive amplified fragment had no complementary binding site with WTB probe, WTB probe exclusively bound to the complementary template region but not the internal competitive amplified fragment, which did not affect the amplification of the internal competitive amplified fragment. Addition of the primer sets of internal reference gene, LEPTIN, massively produce the internal competitive amplified fragments, which helped consuming any excess DNA polymerase and free base fragment and reducing the likelihood of false mismatch, thereby increasing the blocking effect of LNA probe on the wild-type gene. Because this was the same reaction system amplifying the same template, this method was able to quantify the total amount of DNA template and reduce contamination by simplifying the steps of the reaction. The present study analyzed the CT values and fluorescence intensity obtained from the amplification to optimize the WIRE-PCR system by adjusting the final concentrations of primers of the internal reference gene and its probe, the primer for $K R A S$ gene and its probe, and the LNA in the reaction system as follows: $500 \mathrm{nM}$ of the primer sets and $100 \mathrm{nM}$ of the fluorescent probe of the internal reference gene; $500 \mathrm{nM}$ of the primer sets and $250 \mathrm{nM}$ of the fluorescent probe of the KRAS gene; and $500 \mathrm{nM}$ of the LNA probe. Subsequent evaluation of the blocking effect on the common DNA quantity in the clinical sample under the optimized reaction system showed that when the wild-type templates ranged from $50 \mathrm{ng}$ to $150 \mathrm{ng}$, the WTB probe effectively blocked the amplification of the wild-type template in the reaction system. Subsequent detection of the trace amount of mutant KRAS in the 50 colorectal biopsies of the patients with suspected colorectal cancer showed that $36 \%$ of the specimens had mutant KRAS, with the mutation rate ranging from 18.6 to $64.2 \%$.

In summary, the constructed internal competitive amplified fragment improved the detection of trace amount of mutant KRAS by WTB in a real-time fluorescence-based quantitative detection assay. Series optimization in primer concentrations, fluorescent probe concentrations, and LNA concentration effectively block the wild-type DNA templates of the specimens used in the PCR system, which in turn, effectively enriched the mutant gene. The resulted showed the sensitivity is as low as single base pair level and completely inhabited WT-alleles of KRAS. Among the 50 colorectal biopsies collected during colonoscopy, the mutation rate of detected trace amount of mutant KRAS was $36 \%$. The wire PCR in the present study was highly sensitive and specific, easily operated and inexpensive method compared to the direct sequencing approach. It could be extensively used to monitor gene mutation in clinical practice and provide references for tumor monitoring and individualized drug therapies.

\section{Acknowledgements}

This work was supported in part by grants from the National 863 Program of China (no. 2013AA020204), and the Scientific Foundation of Chongqing (no. CSTC2014YYKFA110029; CSTC2015JCSF0105;CSTC2015ZDCY-ZTZX0065; CSTC2015SHMS-ZTZXX0001).

\section{References}

1. Ferlay J, Shin HR, Bray F, Forman D, Mathers C and Parkin DM: Estimates of worldwide burden of cancer in 2008: GLOBOCAN 2008. Int J Cancer 127: 2893-2917, 2010.

2. Siegel R, Naishadham D and Jemal A: Cancer statistics, 2013. CA Cancer J Clin 63: 11-30, 2013.

3. Venook AP: Epidermal growth factor receptor-targeted treatment for advanced colorectal carcinoma. Cancer 103: 2435-2446, 2005.

4. Wadlow RC, Hezel AF, Abrams TA, Blaszkowsky LS, Fuchs CS, Kulke MH, Kwak EL, Meyerhardt JA, Ryan DP, Szymonifka J, et al: Panitumumab in patients with KRAS wild-type colorectal cancer after progression on cetuximab. Oncologist 17: 14, 2012

5. Karapetis CS, Khambata-Ford S, Jonker DJ, O'Callaghan CJ, Tu D, Tebbutt NC, Simes RJ, Chalchal H, Shapiro JD, Robitaille S, et al: K-ras mutations and benefit from cetuximab in advanced colorectal cancer. N Engl J Med 359: 1757-1765, 2008.

6. Amado RG, Wolf M, Peeters M, Van Cutsem E, Siena S, Freeman DJ, Juan T, Sikorski R, Suggs S, Radinsky R, et al: Wild-type KRAS is required for panitumumab efficacy in patients with metastatic colorectal cancer. J Clin Oncol 26: 1626-1634, 2008

7. Kranenburg O: The KRAS oncogene: Past, present, and future. Biochim Biophys Acta 1756: 81-82, 2005.

8. De Roock W, Jonker DJ, Di Nicolantonio F, Sartore-Bianchi A, Tu D, Siena S, Lamba S, Arena S, Frattini M, Piessevaux H, et al: Association of KRAS p.G13D mutation with outcome in patients with chemotherapy-refractory metastatic colorectal cancer treated with cetuximab. JAMA 304: 1812-1820, 2010.

9. Anderson SM: Laboratory methods for KRAS mutation analysis. Expert Rev Mol Diagn 11: 635-642, 2011.

10. Lenz HJ: Testing for RAS mutations in patients with metastatic colorectal cancer. Clin Adv Hematol Oncol 12: 48-49, 2014.

11. Oldenburg RP, Liu MS and Kolodney MS: Selective amplification of rare mutations using locked nucleic acid oligonucleotides that competitively inhibit primer binding to wild-type DNA. J Invest Dermatol 128: 398-402, 2008

12. Huang Q, Wang GY, Huang JF, Zhang B and Fu WL: High sensitive mutation analysis on KRAS gene using LNA/DNA chimeras as PCR amplification blockers of wild-type alleles. Mol Cell Probes 24: 376-380, 2010.

13. Dominguez PL and Kolodney MS: Wild-type blocking polymerase chain reaction for detection of single nucleotide minority mutations from clinical specimens. Oncogene 24: 6830-6834, 2005.

14. Huang JF, Zeng DZ, Duan GJ, Shi Y, Deng GH, Xia H, Xu HQ, Zhao N, Fu WL and Huang Q: Single-tubed wild-type blocking quantitative pcr detection assay for the sensitive detection of codon 12 and 13 KRAS mutations. PLoS One 10: e145698, 2015.

15. Di Giusto DA and King GC: Strong positional preference in the interaction of LNA oligonucleotides with DNA polymerase and proofreading exonuclease activities: Implications for genotyping assays. Nucleic Acids Res 32: e32, 2004.

16. You Y, Moreira BG, Behlke MA and Owczarzy R: Design of LNA probes that improve mismatch discrimination. Nucleic Acids Res 34: e60, 2006.

17. Bustin SA, Benes V, Garson JA, Hellemans J, Huggett J, Kubista M, Mueller R, Nolan T, Pfaffl MW, Shipley GL, et al: The MIQE guidelines: Minimum information for publication of quantitative real-time PCR experiments. Clin Chem 55: 611-622, 2009.

18. Custodio A and Feliu J: Prognostic and predictive biomarkers for epidermal growth factor receptor-targeted therapy in colorectal cancer: Beyond KRAS mutations. Crit Rev Oncol Hematol 85: 45-81, 2013.

19. Kimura T, Okamoto K, Miyamoto H, Kimura M, Kitamura S, Takenaka H, Muguruma N, Okahisa T, Aoyagi E, Kajimoto M, et al: Clinical benefit of high-sensitivity KRAS mutation testing in metastatic colorectal cancer treated with anti-EGFR antibody therapy. Oncology 82: 298-304, 2012. 
20. Smit VT, Boot AJ, Smits AM, Fleuren GJ, Cornelisse CJ and Bos JL: KRAS codon 12 mutations occur very frequently in pancreatic adenocarcinomas. Nucleic Acids Res 16: 7773-7782, 1988.

21. Eberhard DA, Johnson BE, Amler LC, Goddard AD, Heldens SL, Herbst RS, Ince WL, Jänne PA, Januario T, Johnson DH, et al: Mutations in the epidermal growth factor receptor and in KRAS are predictive and prognostic indicators in patients with non-small-cell lung cancer treated with chemotherapy alone and in combination with erlotinib. J Clin Oncol 23: 5900-5909, 2005.

22. Lievre A, Bachet JB, Boige V, Cayre A, Le Corre D, Buc E, Ychou M, Bouché O, Landi B, Louvet C, et al: KRAS mutations as an independent prognostic factor in patients with advanced colorectal cancer treated with cetuximab. J Clin Oncol 26: 374-379, 2008.

23. Itonaga M, Matsuzaki I, Warigaya K, Tamura T, Shimizu Y, Fujimoto M, Kojima F, Ichinose M and Murata S: Novel methodology for rapid detection of KRAS mutation using PNA-LNA mediated loop-mediated isothermal amplification. PLoS One 11: e151654, 2016
24. Dono M, Massucco C, Chiara S, Sonaglio C, Mora M, Truini A, Cerruti G, Zoppoli G, Ballestrero A, Truini M, et al: Low percentage of KRAS mutations revealed by locked nucleic acid polymerase chain reaction: Implications for treatment of metastatic colorectal cancer. Mol Med 18: 1519-1526, 2013.

25. Chen D, Yang Z, Xia H, Huang JF, Zhang Y, Jiang TN, Wang GY, Chuai ZR, Fu WL and Huang Q: Enhanced specificity of TPMT*2 genotyping using unidirectional wild-type and mutant allele-specific scorpion primers in a single tube. PLoS One 9: e91824, 2014.

26. Yuryev A: PCR primer design using statistical modeling. Methods Mol Biol 402: 93-104, 2007.

27. Wangkumhang P, Chaichoompu K, Ngamphiw C, Ruangrit U, Chanprasert J, Assawamakin A and Tongsima S: WASP: A Web-based Allele-Specific PCR assay designing tool for detecting SNPs and mutations. BMC Genomics 8: 275, 2007. 